\title{
Tactile Sensing Arrays for Humanoid Robots
}

\author{
Ravinder S.Dahiya ${ }^{1,2}$, Maurizio Valle ${ }^{2}$, Giorgio Metta ${ }^{1,2}$ \\ 1. Italian Institute of Technology \\ 2. University of Genova \\ Genova, Italy \\ \{Ravinder.Dahiya, Maurizio.Valle\}@unige.it; pasa@liralab.it
}

\author{
Leandro Lorenzelli \\ Fondazione Bruno Kesslar -IRST \\ Trento, Italy \\ lorenzel@itc.it
}

\begin{abstract}
The development of robots capable of operating in unstructured environments or intended to substitute for man in hazardous or inaccessible environments, demands the implementation of sophisticated sensory capabilities, far beyond those available today. In this regard, the development of tactile sensors is one of the key technical challenges in advanced robotics and minimally invasive surgery. In this work we present arrays of 'taxels' (tactile elements) which will be placed on the distal phalange of the humanoid robot in our lab. We present two different designs and implementations. In the first one, microelectrode arrays (MEAs) of 32 elements, with $1 \mathrm{~mm}$ center to center distance, have been designed. The taxel is implemented by putting the sensing material (piezoelectric polymer film of PVDF-TrFE) on a microelectrode. Each taxel is intended to be used as an extended gate of an FET (external to the chip); the taxel collects the charge/voltage generated, as consequence of the applied stress, on the deposited piezoelectric polymer film (i.e. the extended gate itself). The second design and implementation integrates, both the taxels array and the FET devices, on the same silicon die.
\end{abstract}

Index Terms - Piezoelectric polymer-FET, MEA, Tactile sensing array,

\section{INTRODUCTION}

With the movement of robots from structured environment of manufacturing plant into the everyday world that people inhabit, research in the area of robotics has also shifted from industrial to humanoid robotics. An important issue for the humanoid robots is the way they interact with the environment. Real-world objects exhibit rich physical interaction behaviors on touch. These behaviors depend on how heavy and hard the object is when hold, how its surface feels when touched, how it deforms on contact and how it moves when pushed etc. The information about real world objects e.g. differences in shapes can also be obtained with vision by moving robots in all directions around the object. But, it is not always possible to move the robot around the object. Whereas, the same can be done easily and also faster with touch sensing.

Whereas, touch sensing is the process of detection and measurement of a contact force at a defined point, tactile sensing is the process of detection and measurement of the spatial distribution of forces perpendicular to a predetermined sensory area and the subsequent interpretation of the spatial information. In addition to robotics, other potential application

The work presented in this paper has been in part supported by the ROBOTCUB project (IST-2004-004370), funded by the European Commission through the Unit E5 -Cognitive Systems. areas of touch sensing are agriculture, food processing, biomedical, entertainment, future domestic and service industries, military (maneuver in darkness) and rehabilitation.

Cue from the human tactile sensing system can be helpful in bringing the level of tactile sensitivity and acuity that humans possess, to the manipulators and to other human/machine interfaces. A general purpose robotic tactile system, in addition to being cost effective, should possess the following characteristics [1]-[3]:

- $\quad$ A large number of taxels; typical estimated range should be between 25- 256 elements.

- Human like spatial sensitivity viz. $1 \mathrm{~mm}$

- Sensitivity to forces spanning from $\operatorname{lgmf}(0.01 \mathrm{~N})$ to $1000 \mathrm{gmf}(10 \mathrm{~N})$ with incremental force resolution of $1 \mathrm{~g}$.

- $\quad$ Discrete taxel response bandwidth of $1000 \mathrm{~Hz}$.

A reasonable response linearity and negligible hysteresis are also desired. The above specifications can be used as a starting point.

Although high quality cameras (used for visual sensing in robots) are readily available in market, it is difficult to find something similar for tactile sensing. Touch sensors using different transduction mechanisms realized in the past are mainly discrete touch sensor elements and provide only limited information like touch or no touch. One such discrete element touch sensor based of Hall Effect was used earlier on one of the humanoid robots in our lab [4]. Relative merits, limitations, performance and reviews of earlier technologies for the tactile sensing have been reported in [3, 5]. For object recognition and to have human like touch sensitivity, a large number of taxels are required. Use of a large number of discrete touch sensor elements would increase the number of interconnections and also the electrical interference. Besides these problems, a limited space on the robot finger makes this approach impractical. These issues can be addressed by way of miniaturization.

In this paper, we present an approach for the development of robotic tactile sensing system by integrating "smart materials" like piezoelectric polymers with the Integrated Circuits (ICs). Fig. 1 shows the proposed tactile sensing system. The integration of sensing elements with electronics on silicon will reduce electrical interference thereby 
improving the $\mathrm{S} / \mathrm{N}$ ratio. This will also help in reducing the wiring complexity, a key problem in robotics. We report the progress toward realizing an array of tactile sensors by direct coupling of piezoelectric polymers thin films to MEAs realized on silicon die. This is further followed by the second design, which is based on the development of MEAs operated as extended gates of FETs integrated on the same chip.

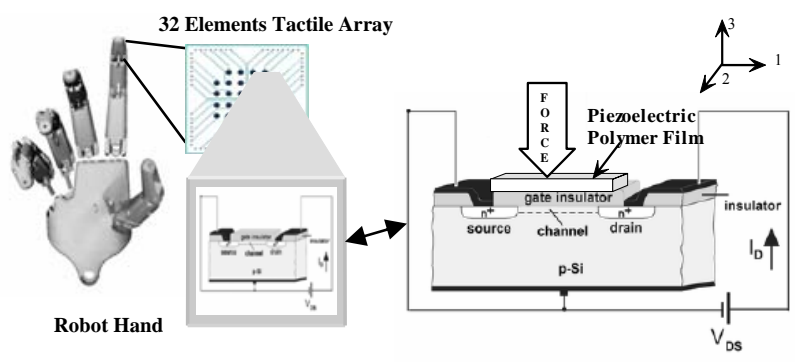

Fig.1. Proposed tactile sensing system. For simplicity, the force is shown in the thickness direction. In general, the polymer response is different for forces in different directions.

In the following sections, we present the principle of operation, modeling and analysis of the sensing element, design and fabrication of devices and the future direction.

\section{PRINCIPLE OF OPERATION}

A piezoelectric film working in the generating mode, gives a charge/voltage output when a mechanical input is applied. The generated charge/voltage is proportional to the applied stress. Thus, if the polysilicon gate of a MOSFET is replaced by a piezoelectric polymer film, the charge (and hence the voltage) generated due to the stress applied on the piezoelectric polymer film controls the charge in the MOSFET channel. In other words, the charge in the channel is modulated by the applied mechanical stress. The signal is amplified by the MOSFET and is then further processed by electronic circuitry.

A similar approach to develop ultrasonic sensors was reported by Swartz et al. [6]. Epoxy adhered PVDF polymer was used on the extended gates in their acoustic sensor. In addition of having good electromechanical properties, PVDFTrFE used in our tactile sensor is easy to deposit on the silicon. General parameters of PVDF and PVDF-TrFE are given in Table 1.

Due to economic reasons, changing the oxide layer thickness, and other dimensions of the devices, by modifying a standard fabrication process is not always possible. The ease with which PVDF-TrFE can be spin coated on the silicon die, provides us with an alternative method to control the capacitance of the polymer film with respect to the oxide and other capacitances of backing material (silicon in our case) and hence to optimize the sensitivity of the transducer.
TABLE I.

PROPERTIES OF PIEZOELECTRIC POLYMERS

\begin{tabular}{|l|c|c|}
\hline Parameter & PVDF $^{\mathbf{a}}$ & PVDF-TrFE $^{\mathbf{a}}$ \\
\hline Density, $\rho\left(\mathrm{Kg} / \mathrm{m}^{3}\right)$ & 1780 & 1880 \\
\hline Longitudinal Velocity, $v(\mathrm{~m} / \mathrm{s})$ & 2200 & 2400 \\
\hline Acoustic Impedance, $Z(\mathrm{MRayl})$ & 3.92 & 4.32 \\
\hline Clamped Dielectric Constant, $\varepsilon_{33}^{S}$ & 5.0 & 4.0 \\
\hline Dielectric Loss Tangent, tan $_{e}$ & 0.25 & 0.12 \\
\hline Mechanical quality factor, $\mathrm{Q}_{\mathrm{m}}$ & 13 & 25 \\
\hline Thickness coupling factor, $\mathrm{k}_{\mathrm{t}}$ & 0.15 & 0.30 \\
\hline
\end{tabular}

${ }^{a}$ From [7]

\section{MODELLING AND ANALYSIS}

A piezoelectric film produces charge/voltage due to the applied stress. The electromechanical behavior of piezoelectric materials can be represented by following mathematical relations [8]:

$$
\begin{aligned}
& T_{3}=c_{33}^{D} S_{3}-h_{33} D_{3} \\
& E_{3}=-h_{33} S_{3}+\beta_{33}^{S} D_{3}=-h_{33} S_{3}+\frac{D_{3}}{\varepsilon}
\end{aligned}
$$

Where $T_{3}, S_{3}, D_{3}, E_{3}, c_{33}^{D}, h_{33}$ and $\varepsilon$ are the stress, strain, electric displacement, electric field, elastic constant, piezoelectric constant and permittivity respectively. The subscripts give the direction of polarization and the direction of applied force. In our case, the polarization is along 3direction (i.e. along thickness). To keep the analysis simple, the force is also assumed to be in same direction. The performance of the piezoelectric polymer under various conditions of polymer thicknesses, type and thickness of the substrate etc. can be analyzed by using its equivalent circuit. This also helps in optimizing the design of tactile sensing systems. The SPICE implementation of the equivalent circuit for piezoelectric polymer operating in the receiving/sensing mode is presented in [9]. The equivalent circuit of proposed tactile sensing system is obtained by adding the SPICE model of MOSFET at the electrical output of the piezoelectric polymer [10]. The equivalent circuit of a piezoelectric polymer-FET tactile sensing element developed with PSpice is shown in Fig. 2.

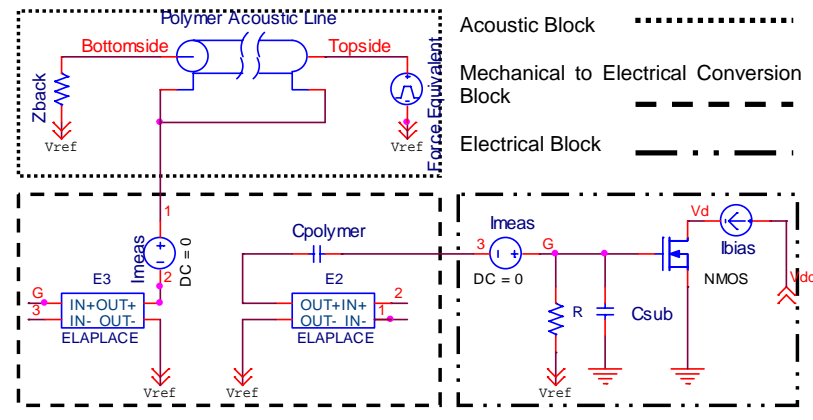

Fig 2. SPICE model of piezoelectric polymer in the sensing mode [10]. 
In this figure the transmission line represents mechanical equivalent of acoustic transmission in piezoelectric polymer, mechanical to electrical conversion is represented by controlled sources and the electrical part is represented by the electrical section.

Using the supplier's data, (1), (2) and the mathematical relations given in [8], open circuit voltage generated $\left(V=d_{33} * F / C_{\text {polymer }}\right.$ ) by a $50 \mu \mathrm{m}$ thick polymer should be nearly one volt, when a $0.01 \mathrm{~N}$ (one gram) force is applied on one taxel (area $0.196 \mathrm{~mm}^{2}$ ) of MEA. The simulated electrical open circuit response of the piezoelectric polymer and the piezoelectric polymer-FET sensing element, when a step of $0.01 \mathrm{~N}$ is applied at the force input terminal, are shown in fig 3 and Fig. 4. The equivalent of force is applied at one end of the transmission line and the other end of the line is terminated with the equivalent of acoustic impedance of silicon.

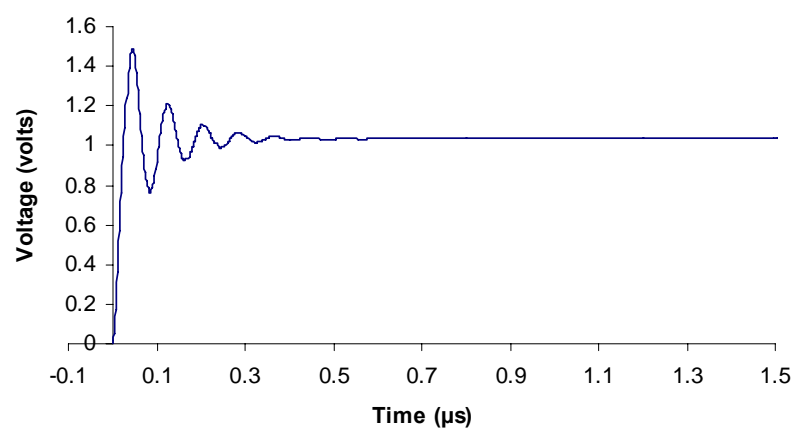

Fig. 3. Transient response of piezoelectric polymer (at node 3 in Fig 2)

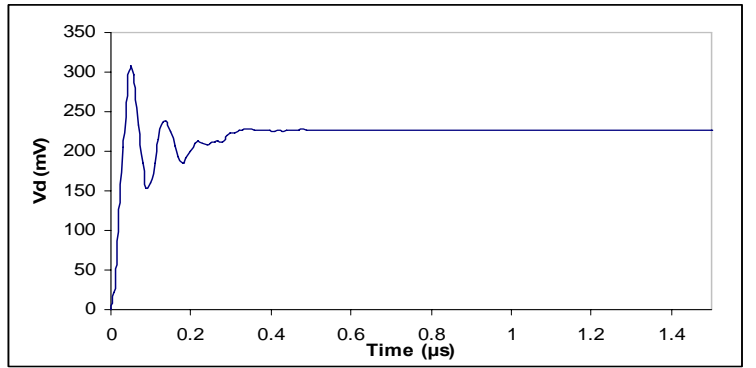

Fig 4: Voltage output of piezoelectric polymer-FET sensing element i.e. $V_{D}$, at the drain terminal of NMOS in fig 2 [9].

It can be noticed that, due to the presence of substrate capacitance $C_{s u b}$ on the chip, the output of piezoelectric polymer-FET sensing element in fig 4 is much lower than that of piezoelectric polymer in Fig. 3. Thus, in order to have maximum voltage at the gate terminal of the FET device, the substrate capacitance of extended gate and the FET device capacitances should be comparable or less than the Cpolymer. At low frequencies the piezoelectric polymer can be approximately represented by a voltage source in series with the capacitance Cpolymer.

\section{DESIGN AND FABRICATION}

The integration of the taxels array and the active devices demands for a fabrication process both technologically reliable and economically suitable to be integrated with different technologies, such as Al-microelectrode fabrication and piezoelectric polymeric layer deposition.

As a first step towards implementation of the touch sensor we have designed and developed test structure based on 32 elements microelectrode arrays at FBK-IRST. One such test structure devoted to characterize the polymeric material and to perform the electrical/mechanical tests to refine the read out electronics is shown in fig 5(a). To study the electrical response of different taxels PVDF-TrFE polymer (supplied by Piezotech) with 25, 50 and $100 \mu \mathrm{m}$ have been deposited using the steps mentioned in table II. The front and backside of the MEA after depositing polymer is shown in fig 5(b). The typical output from one of the taxels obtained after depositing polymer film on the MEA is shown in fig 6.

The fabrication of MEA is implemented on a fuse silica quartz substrate a $\mathrm{Al}: \mathrm{Si} 1 \% / \mathrm{Ti} / \mathrm{TiN}$, respectively of $410 / 30 / 140 \mathrm{~nm}$ thick, low resistance multilayer for both microelectrodes and electrical connections. The TiN top-layer has been introduced to guarantee a low contact resistance to the final $\mathrm{Au} / \mathrm{Cr}(5 / 150 \mathrm{~nm})$ seed-layer. The metal wires passivation has been guaranteed by a $\mathrm{SiO}_{2} / \mathrm{Si}_{3} \mathrm{~N}_{4}(20 / 210 \mathrm{~nm})$ layer deposited by plasma enhanced chemical vapor deposition (PECVD) method. These thicknesses have been chosen to keep the substrate capacitance low and hence to get the maximum of the voltage produced by polymer at the gate terminal. Here, a quartz wafer has been used instead of silicon to minimize the cross talk. Future arrays will be implemented on silicon wafers.

Table II

MAIN FABRICATION PROCESS SEQUENCE

\begin{tabular}{|c|c|}
\hline \multicolumn{2}{|r|}{ Fabrication Process } \\
\hline a. & Substrate: $500 \mu \mathrm{m}$ thick quartz wafer. \\
\hline b. & $\mathrm{A}$ Ti/TiN/Al/TiN multilayer is deposited by sputtering. \\
\hline c. & $\begin{array}{l}\text { This multilayer is patterned by photolithography and plasma dry } \\
\text { etching to form the electrodes, lines and the contact pin zone. }\end{array}$ \\
\hline d. & $\begin{array}{l}\text { A layer of } \mathrm{Si}_{3} \mathrm{~N}_{4}(200 \mathrm{~nm}) \text { is then deposited by PECVD, in order to } \\
\text { insulate the metal lines. }\end{array}$ \\
\hline e. & $\begin{array}{l}\text { Contacts are opened through the } \mathrm{Si}_{3} \mathrm{~N}_{4} \text { layer by plasma } \\
\text { dry etching }\end{array}$ \\
\hline f. & $\begin{array}{l}\text { Evaporation of } \mathrm{Cr} \text { and } \mathrm{Au} \text { is carried out }(5 \mathrm{~nm} \text { and } 150 \mathrm{~nm} \text {, } \\
\text { respectively) }\end{array}$ \\
\hline g. & The Chromium and Gold layers are patterned by wet etching. \\
\hline $\mathrm{h}$ & $\begin{array}{l}\text { Deposition of piezoelectric polymer. The film was deposited using } \\
\text { epoxy adhesive on the MEA, covering all } 32 \text { taxels. The film was } \\
\text { covered with glass slide and the arrangement was again kept under } \\
\text { vacuum to remove air between polymer and MEZ and to ensure } \\
\text { uniform thickness of the adhesive. For better adhesion the } \\
\text { arrangement was kept at } 65 \text { degrees for thirty minutes. }\end{array}$ \\
\hline
\end{tabular}




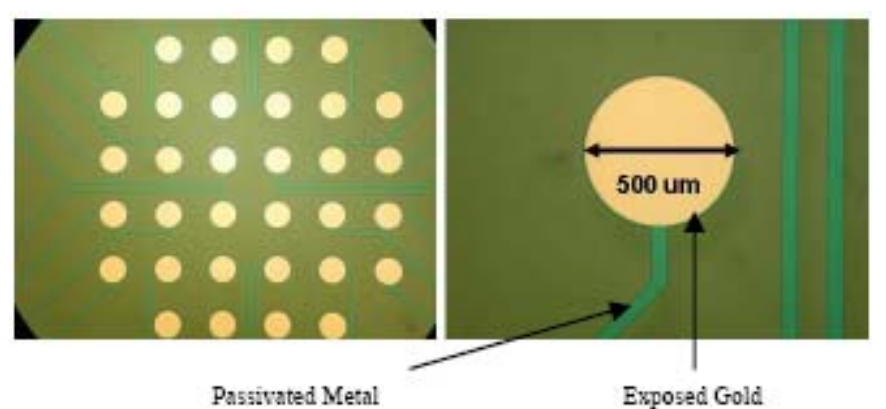

(a)

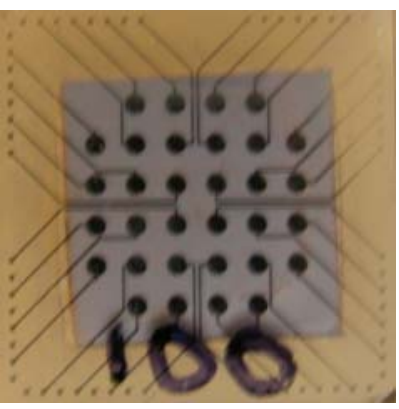

Back of MEA
Piezoelectric polymer film

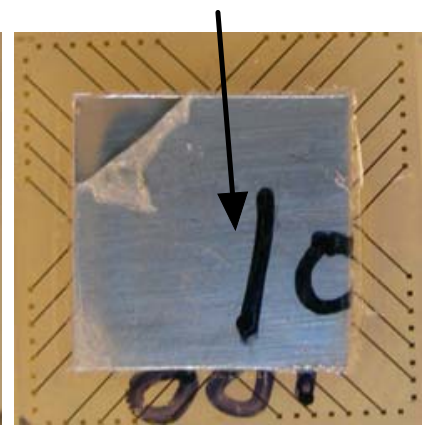

Top of MEA (b)

Fig.5 a) MEA for the extended gate-FET approach. Chip dimension is $1 \mathrm{~cm} \mathrm{x}$ $1 \mathrm{~cm}$. and diameter of the taxels is $500 \mu \mathrm{m}$ (b) Back and top sides of MEA with $100 \mu \mathrm{m}$ polymer covering all the electrodes. A general purpose protecting tape can also be seen on top of polymer.

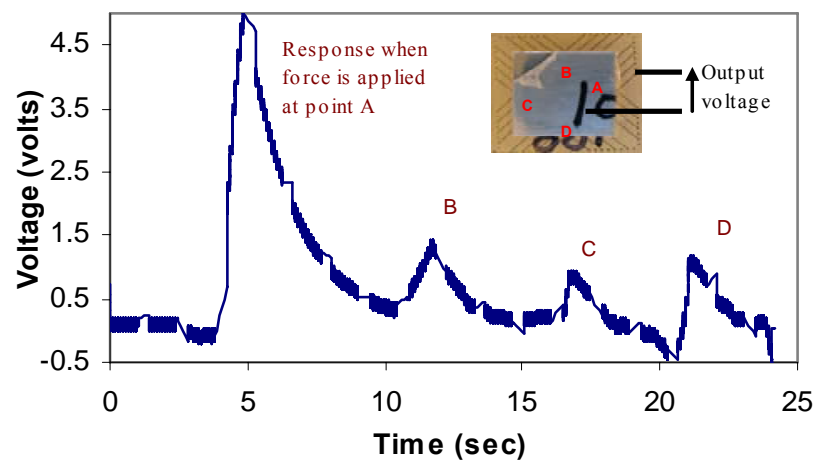

Fig 6: The output from the MEA with a $100 \mu \mathrm{m}$ epoxy adhered polymer film. The force was applied on the taxel at point ' $A$ ', which is also connected with the measuring instrument. The response of taxel at $\mathrm{A}$, when force was applied at points $\mathrm{B}, \mathrm{C}$, and $\mathrm{D}$ is also shown. Thus, the point at which force is applied can be clearly differentiated.

In the second implementation, as shown in fig. 7, the taxels array and the MOSFETs are integrated on the same silicon die. The developed chip consists of an array of $25 \mathrm{n}$ MOS elements (with $\mathrm{W} / \mathrm{L}=300$ ) where the exposed dielectric gate and metal ring have been connected in a diode configuration in order to optimize the device versus read-out

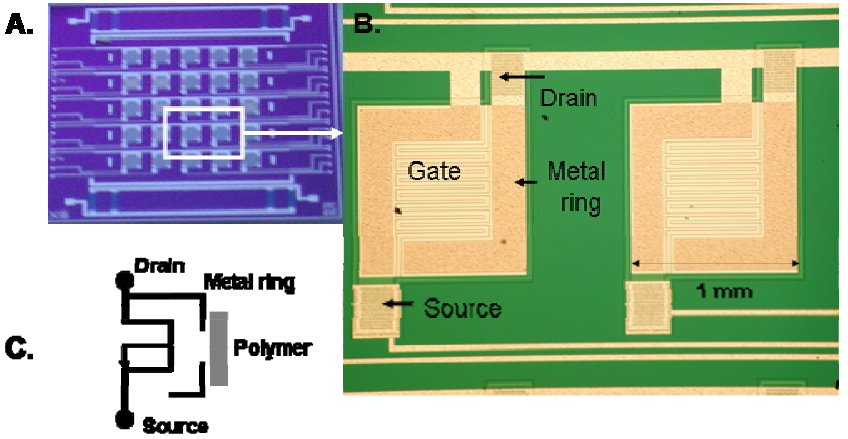

Fig. 7: A) Chip prototype $(1 \mathrm{~cm} \times 1 \mathrm{~cm}$ die size $)$ B) Enlarged photograph of the n-MOS sensor cell; C) Scheme of the electrical configuration

connections. The reference fabrication process for this second design is the n-MOS technological module of a non standard 4- $\mu \mathrm{m} \mathrm{Al}$ gate $\mathrm{p}$-well ISFET/CMOS technology [11]. The main features are a $\mathrm{Si}_{3} \mathrm{~N}_{4} / \mathrm{SiO}_{2}$ double layer as a gate dielectric and a second metal for implementing microelectrodes and metalrings. In order to achieve a "Piezoelectric polymer - FET" working mode, piezoelectric polymeric layer (PVDF-TrFE) will be spun onto the whole wafer surface. These devices are now under testing and the dimension of final device and package would be chosen in accordance with the space available on the distal phalange of the humanoid robot of our lab.

\section{CONCLUSION AND FUTURE WORK}

An approach for development of a new tactile sensor system for humanoid robots is presented along with the preliminary designs to test the piezoelectric polymers separately and with FET. The distributed forces and contact points, obtained from the array of sensors can be used to find the physical properties like slip, shape, texture etc [1]. The fact that the frequency response of piezoelectric material changes with the contact material can be exploited not only to distinguish different materials but also to find the hardness/softness of the material. In this sense, the tactile sensing array is expected to have an edge over the vision sensing.

The SPICE model of piezoelectric polymer in sensing mode is presented for a lossless case. Simulated output is found to be in agreement with the theoretical results. As reported earlier [6], the piezoelectric polymers have more losses than piezoceramics. The SPICE model presented in this work can be improved by introducing the complex values of piezoelectric constant, dielectric constant and the elastic constant. The SPICE model would help in the optimizing the design of sensor system and the fabrication sequence or the polymer thickness can be modified accordingly in the next stage. Future work will involve the study of electrical output of a single taxel as well as that of the array, by applying forces 
with different magnitudes and frequencies. For this purpose, a test set up is being developed.

Piezoelectric polymers in general, respond to both mechanical and temperature changes. Since the humanoid robots are expected to work at different environmental conditions, it is necessary to ensure that the sensor responds only when touched or when the robot touches something. The same argument applied to other potential applications like biomedical applications. Thus, the pyroelectric effect of the polymer needs to be compensated. This can be done either by observing the response of piezoelectric polymer to changes in temperatures only and then mathematically compensating it in the overall response of the sensor or by using some other material that is more responsive to mechanical changes than temperature changes. The effect of temperature variations can also be compensated by introducing a temperature sensing device on the chip itself and hence keeping the temperature of polymer constant. In the second design of the chip this technique would be implemented. Also, in the next stage of the fabrication process, a process for array of n-MOS devices without metal gate will be realized and the complex circuits including both sensors and read-out will be accommodated on the same chip.

\section{REFERENCES}

[1] R.D. Howe, "Tactile sensing and control of robotic manipulation", Journal of Advanced Robotics, 8(3): 245-261, 1994.

[2] L. D Harmon. "Automated tactile sensing," International Journal of Robotics Research 1(2):3-31, 1982.

[3] P. Dario, Danilo D. Rossi, "Tactile sensors and the gripping challenge", IEEE Spectrum, 46-52, August 1985.

[4] L. Jamone, Giorgio Metta, F. Nori, G. Sandini, "James: A humanoid Robot Acting over an Unstructured World," Proceedings of the $6^{\text {th }}$ IEEERAS Int. Conf. on Humanoid Robots, Genoa, pp 143-150, 2006

[5] M.H. Lee and H.R. Nicholls "Tactile sensing for mechatronics - a state of art survey", Mechatronics, 9(1), October 1999.

[6] Robert G. Swartz, James D. Plummer, "Integrated silicon-PVF2 acoustic transducer arrays," IEEE transactions on Electron Devices, Vol. 26, No-12, p 1920-32, Dec 1979.

[7] Lewis F. Brown, "Design Considerations for Piezoelectric Polymer Ultrasound Transducers," IEEE Trans. Ultrasonics, Ferroelectrics and Freq. Control, Vol. 47, no. 6, 1377-1396, Nov 2000

[8] ANSI/IEEE Standard of Piezoelectricity, 176-1987.

[9] Ravinder S. Dahiya, M. Valle, G. Metta, L. Lorenzelli Cristian Collini, "Tactile sensing arrays for humanoid robot," PRIME 2007, $3^{\text {rd }}$ Conf. on PhD research on Microelectronics and Electronics, 2007 (in press)

[10] Ravinder S. Dahiya, M. Valle, G. Metta, L. Lorenzelli, "POSFET Based Tactile Sensor Arrays," 14th IEEE International Conference on Electronics, Circuits and Systems 2007 (unpublished).

[11] S. Martinoia, et. al, "Development of ISFET array-based Microsystems for bioelectrochemical measurements of cell populations," Biosensors \& Bioelectronics, 16, p 1043-1050, 2001. 